\title{
Mechanism of supported liquid membrane degradation: emulsion formation
}

\author{
A.M. Neplenbroek, D. Bargeman and C.A. Smolders \\ University of Twente, Department of Chemical Technology, P.O. Box 217, Enschede (Netherlands)
}

(Received July 13, 1990; accepted in revised form March 27, 1991)

\begin{abstract}
A new hypothesis for the degradation mechanism of supported liquid membranes is advanced: emulsion formation induced by shear forces. Experiments show that the removal of LM-phase from the membrane depends in the molecular structure of the carrier and the type of solvent. The instability of SLMs is regulated by the presence of counter-ions in the same way as in the case of emulsion stability: a decrease in salt concentration in the aqueous phases and an increase in flow velocity of these phases parallel to the membrane surface both lead to an increase in instability of the liquid membrane, while emulsion formation is stimulated by these circumstances.
\end{abstract}

Keywords: coupled, facilitated transport; liquid membranes; supported liquid membranes; liquid membrane stability

\section{Introduction}

One of the main problems in using the supported liquid membrane (SLM) technique in practical situations is the lack of stability of the liquid membrane. Therefore the stability of these membranes should be enhanced drastically. Because there are still many unknowns about the reasons for SLM-instability, further investigations are necessary. In Ref. [1] a first impulse to this study was given. In this article the results of a more systematic search into the factors influencing the stability will be described to get a better knowledge of the mechanism of SLM-degradation.

Correspondence to: D. Bargeman, University of Twente, Department of Chemical Technology, P.O. Box 217, Enschede, Netherlands.
The results given in Ref. [1] showed that the stability of SLMs depends very much on the type of solvent used, on the molecular structure of the carrier and also on the composition of the aqueous phase. In this paper two aspects of SLM-instability will be studied further: to begin with the leakage behaviour of SLMs with decanol as solvent gets extra attention. Secondly the long term permeability behaviour of two SLMs with different carriers will be investigated.

A clear correlation is found between the instabilities which are observed for SLMs and the degree of emulsion formation between the same aqueous phases and the LM-phases. This leads to the hypothesis that the instability of SLMs is caused by the formation of emulsions. To test this hypothesis the stability of SLMs is studied by flowing aqueous phases with similar com- 
positions along the feed and strip-side of the membranes to exclude any effect of osmotic pressure differences. It will appear that following this line of investigations the instability problem can be approached more directly.

Furthermore a number of quantities affecting emulsion formation and emulsion stability are measured and compared with SLM-instability effects. A model describing the mechanism of SLM-degradation will be proposed.

\section{Experimental set-up}

In this paper two experimental routes will be followed: on the one hand measurements are done in which aqueous phases flow parallel to a membrane and on the other hand experiments are performed in which the formation of emulsions between the LM-phase and these aqueous phases is studied.

\section{SLM-instability measurements}

For the experiments in which the aqueous phases flow parallel to the SLMs, the permeability apparatus as described before was used [2]. The chemicals that are used as components of the LM-phase are the same as those mentioned in Ref. [1] and the membranes are prepared in the same way as described there. Celgard $^{\circledR} 2500$ (microporous polypropylene from Celanese; thickness: $25 \mu \mathrm{m}$; porosity $45 \%$; pore dimensions $0.04 \mu \mathrm{m}$ ) has been used as support for all the experiments described in this paper.

\section{Membrane failure for decanol as membrane phase}

In these experiments the membrane phase consists of decanol without carrier. The starting compositions of the aqueous phase are: 0.004 $\mathrm{M} \mathrm{NaNO}_{3}$ ("feed F") and 4.0 $\mathrm{M} \mathrm{NaCl}$ ("stripping phase S"). Before starting the experiment the aqueous phases are saturated with decanol by adding an excess of $0.5 \mathrm{ml}$ decanol per liter of aqueous phase under stirring. An organic phase in contact with the aqueous phase remained visible during the experiment because of the excess of decanol added.

The membrane was in contact with a nonstreaming water phase or the water phase was passed along with a flow velocity of $5.5 \mathrm{ml} / \mathrm{sec}$. In case of different flow velocities, we compensated for the resulting pressure difference by a correction in height of the buffer vessels. Samples of the feed are taken periodically (each half hour) and the chloride and nitrate content was determined with HPLC. From this analysis the occurrence of an extra transport resulting from membrane leakage could be determined.

The masses of the membranes were determined before starting and after finishing the experiment (after $7 \mathrm{hr}$ ). From the difference the percentage decanol removed from the microporous support was obtained.

\section{Long term permeation}

The long term permeation behaviour was studied for SLMs with 0 -nitrophenyloctylether (o-NPOE) as solvent and using as carriers: tetraoctylammonium bromide (TeOA) and trioctylmethyl ammonium chloride (TOMA) respectively $(0.2 \mathrm{M})$. At the beginning of the flux measurements the aqueous phases, which flowed along the membrane day and night with a flow velocity of $5.5 \mathrm{ml} / \mathrm{sec}$, had a composition of $0.004 M \mathrm{NaNO}_{3}$ (F) and $4.0 \mathrm{M} \mathrm{NaCl}$ (S). The nitrate and chloride content was measured as a function of time by analyzing samples, which were taken periodically from the feed, using HPLC. The nitrate flux was calculated from the slope of the curve giving the nitrate concentrations as a function of time.

After 3 or 4 days both water phases were replaced by fresh starting solutions, after which the flux was determined again. After ca. 8 days the presence of tiny gasbubbles became visible 
at the surface of the membrane with $\mathrm{TeOA}$ as carrier. These bubbles were removed by tapping.

From the increase in chloride content in the feed, and the calculation of the counter transport factor (CTF: the ratio of the decrease in nitrate content and the increase in chloride concentration in the feed) as a function of time as given in Ref. [1], the occurrence of direct leakage from stripping phase to feed could be determined.

\section{Removal of LM-components}

During these measurements feed and stripping phase consisted of exactly the same compositions when flowing along the membrane surfaces for some time. The salt concentrations in the aqueous phases are varied from $0 M$ to 4 $M \mathrm{NaCl}$. In one series of experiments we used aqueous phases with $\mathrm{NaClO}_{4}$, with a concentration varying from $10^{-4}$ to $10^{-2} \mathrm{M}$. Furthermore the water flow velocities and the duration of the experiments are varied.

\section{Solvent removal}

In these measurements the support is filled with a pure solvent. The amount of LM-phase removed from the support was calculated by determining the weight of the membranes before the experiment was started and by weighing the centres of the membranes after the measurements were finished, analogous to the experiments described in Ref. [1].

\section{Carrier removal}

The amount of carrier removed from the central area of the membranes was determined spectrophotometrically as described in Ref. [1]. In those cases where $\mathrm{NaClO}_{4}$ ions were present in the aqueous phases the membranes were soaked in a $4 \mathrm{M} \mathrm{NaNO}_{3}$ solution after the experiment. This was done to achieve an ion-exchange between the $\mathrm{ClO}_{4}^{-}$(bound to the carrier) and $\mathrm{NO}_{3}^{-}$. This extra step in the analysis is necessary since, according to the Hofmeister series, the $\mathrm{ClO}_{4}^{-}$ion is more lipophilic (i.e. more strongly complexed) than the 4-(2-pyridylazo) resorcinol ("PAR") ion with which the carrier should be complexed to carry out the analysis. Also the carrier concentrations in the aqueous phases were determined spectrophotometrically after extraction of these phases with chlorobenzene.

\section{Emulsion formation and stability}

\section{Preparation of emulsions}

Emulsions of an LM-phase in a aqueous phase are prepared in three different ways:

- By stirring: three droplets of an organic phase are added to $50 \mathrm{ml}$ salt solution of varying concentrations in a beaker. This mixture is stirred during 3 min with a stirrer (Janke \& Kunkel) at a speed of $250 \mathrm{rpm}$. Samples of these mixtures are put into a spectrophotometer tube and the transmission is determined as a function of time.

- By shaking: $1 \mathrm{ml} \mathrm{LM}$-phase and $3 \mathrm{ml}$ water phase are added to a spectrophotometer tube. This tube is plugged and shaken by hand rather strongly. After $5 \mathrm{~min}$ the transmission of the aqueous phase is determined.

- By ultrasonic vibration: approximately 0.05 $\mathrm{g}$ of the LM-phase is added to $500 \mathrm{ml}$ doubledistilled water in a beaker. This beaker is vibrated ultrasonically for $45 \mathrm{~min}$ in the water bath of a Bransonic (50-55 kHz). Hereafter 1 $\mathrm{ml}$ samples of the emulsion are added to spectrophotometer tubes containing $5 \mathrm{ml}$ of salt solutions of varying concentrations and mixed. The transmission of these emulsions then is determined as a function of time. In all cases the transmission of the emulsions in the spectrophotometer tubes is determined with a Spectronic 20 at a wavelength of $500 \mathrm{~nm}$.

For the emulsions prepared by the ultrasonic vibration method we also measured the particle size distribution in the emulsion with a Mal- 
vern Master sizer and the zeta-potential of the particles with a Malvern Zetasizer.

\section{Results and discussion}

\section{Membrane failure for decanol as membrane phase}

It was shown before [1] that membranes with decanol as solvent showed the largest instability effects. This gives the opportunity to investigate the factors influencing this instability within a reasonable period of time. The time needed to reach the point where the membrane showed leakage (CTF larger than 1) and the amount of removal of the LM-phase proved to be independent of:

- the volume of the feed phase (varying from 4.11 to 0.131 );

- the saturation of the aqueous phases with decanol;

- the saturation of decanol with water before filling the support;

- the presence of the carrier TeOA (up to 0.2 $M$ ) in the membrane phase.

Thereupon it was examined whether in particular the flow of one of the aqueous phases (the feed with $0.004 \mathrm{M} \mathrm{NaNO}_{3}$ or the stripping phase with $4.0 \mathrm{M} \mathrm{NaCl}$ ) was responsible for the removal of LM-phase from the support, resulting in chloride leakage. To make the system as simple as possible SLMs with pure decanol were used. At first these experiments were carried out with Accurel as support. Later on Accurel was replaced by Celgard since this latter support showed the same trends in instability effects, but in a shorter time. In agreement with the results of other researchers [3] it was found that the use of Accurel as support leads to substantially more stable SLMs. It appears from our measurements that the first signs of leakage of chloride were observable in a 10 times shorter time for Celgard than for Accurel. Since the relative instability effects were comparable to each other, for practical reasons Celgard was chosen as support. In Fig. 1 the chloride concentration in the feed is given as a function of time for three SLMs, with decanol as LM-phase and Celgard as support, for different flow velocities of feed and stripping phase.

The amounts of LM-phase which are removed from the membranes in these systems by a flow of the aqueous phases during $7 \mathrm{hr}$ are given in Table 1.

From Table 1 it can be concluded that the flow rate at the stripping side (with a high salt concentration) is of minor importance for the amount of LM-phase removed. Furthermore it appears that the larger part of LM-removal results from shearing forces excerted at the feed side having a low salt concentration. These re-

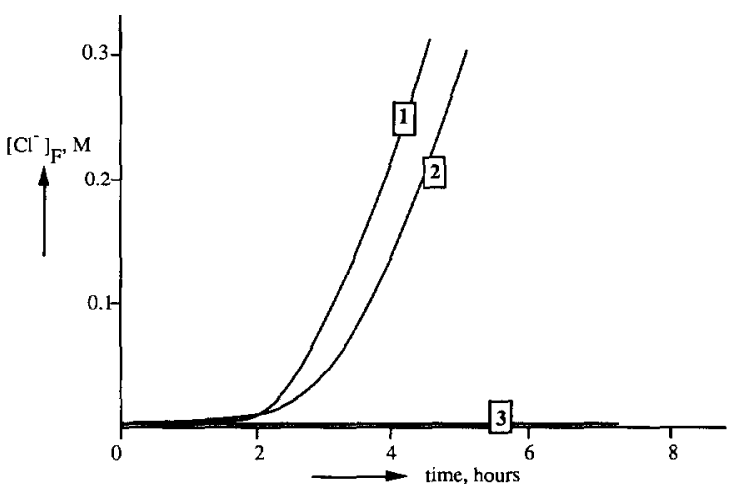

Fig. 1. Influence of flow rate of aqueous phases on chloride leakage through decanol-SLMs; (1) flow rate $\mathrm{F}=$ flow rate $\mathrm{S}=5.5 \mathrm{ml} / \mathrm{sec}$; (2) flow rate $\mathrm{F}=5.5 \mathrm{ml} / \mathrm{sec}$ and flow rate $\mathrm{S}=0 \mathrm{ml} / \mathrm{sec}$; (3) flow rate $\mathrm{F}=0 \mathrm{ml} / \mathrm{sec}$ and flow rate $\mathrm{S}=5.5$ $\mathrm{ml} / \mathrm{sec}$.

\section{TABLE 1}

Influence of aqueous flow rate on LM-removal, after $7 \mathrm{hr}$, from decanol-SLMs

\begin{tabular}{llll}
\hline No. & $\begin{array}{l}\text { Flow rate F } \\
(\mathrm{ml} / \mathrm{sec})\end{array}$ & $\begin{array}{l}\text { Flow rate S } \\
(\mathrm{ml} / \mathrm{sec})\end{array}$ & $\begin{array}{l}\text { LM-removal } \\
(\%)\end{array}$ \\
\hline 1 & 5.5 & 5.5 & 44 \\
2 & 5.5 & 0.0 & 43 \\
3 & 0.0 & 5.5 & 19 \\
\hline
\end{tabular}

$\mathbf{F}=$ feed phase $\mathrm{S}=$ stripping phase. 
sults agree with the leakage behaviour of the SLMs as given in Fig. 1. Especially for those systems where the feed is flowing at $5.5 \mathrm{ml} / \mathrm{sec}$ and where a large amount of LM-phase has been removed, the membranes start to leak within a short time. When the feed does not flow at all a relatively stable SLM is obtained which shows no sign of chloride ion leakage within $7 \mathrm{hr}$. Obviously a short-circuiting between the $F$ and $S$ phases arises at that moment when a certain amount of LM-phase (above 20\%) has been removed from the pores of the membrane.

\section{Long term permeation}

Apart from a possible leakage of SLMs, the decrease in nitrate flux as a function of time is also an important aspect in SLM-stability. It is possible that this behaviour is determined largely by the degree in which the carrier has been removed from the support. To investigate the influence of the structure of the carrier, we measured the long term permeability for membranes using TeOA and TOMA as carriers. It was shown before [1] that the degree to which these carriers were washed out from a membrane phase differed largely. To minimize the influence of the solvent, $o-\mathrm{NPOE}$ was chosen because this solvent gives the most stable membranes as was shown previously.

Based on previous measurements it could be expected that, for a system with TeOA as carrier and Accurel as support, such an investigation would last at least several months before leakage through the membrane would occur. Therefore Celgard was chosen as support to determine the influence of the structure of the carrier on flux decline.

In Fig. 2 the long term permeability of SLMs with TeOA and TOMA is given as a function of time.

During these measurements the countertransport factor (CTF) was also measured. From the results we concluded that the membrane with TeOA as carrier was leaking chlo-

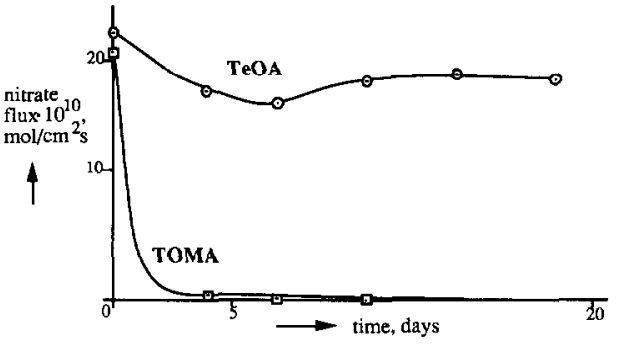

Fig. 2. Long term permeation; flux as a function of time and type of carrier; LM-phase: $0.2 M$ carrier in o-NPOE; support Celgard; feed $0.004 \mathrm{MNaNO}_{3}$; stripping phase 4.0 $M \mathrm{NaCl}$; flow velocity water phases $5.5 \mathrm{ml} / \mathrm{sec}$.

ride ions (CTF>1) from the 4th day on. The membrane with TOMA as carrier showed a leakage of chloride ions for the first time on day 7. Furthermore the degree of chloride leakage was larger for the TeOA-membrane than for the TOMA-membrane. Similar results were obtained from duplicated measurements. It is remarkable that, as already mentioned in Ref. [1], the process of nitrate removal generally continues after leaks in the membrane have appeared. Apparently we are dealing with a restricted occurrence of channels in the membrane, while the larger part of the membrane is still intact and keeps on functioning.

The results given in Fig. 2 show that the membrane with $\mathrm{TeOA}$ as carrier gives a nearly constant flux indicating that we are dealing with a rather stable membrane. The slight decrease in flux during the first 7 days is probably caused by the adsorption of air bubbles, which are present or being formed in the system, onto the membrane surface; the effective contact area between the aqueous phases and the liquid membrane decreases and so does the flux. Removal of these air bubbles after 8 days leads to a slight increase in flux. Air bubble adhesion to the hydrofobic material can be a general problem for the application of SLM-separation processes and the magnitude of this effect will depend on the hydrodynamic conditions.

LM phase removal from the TeOA mem- 
brane is directly confirmed by the result that the membrane shows a chloride-ion leakage after a reasonable short time (4 days). $\mathrm{Ob}$ viously LM-phase is being removed at some loci to such an extent that direct channels of aqueous phase can be formed and the flow between F- and S-side is short-circuited. The fact that despite loss in carrier concentration (see Ref. [1]) and channeling the nitrate flux is rather constant during a much longer time than 4 days leads to the following tentative conclusions:

- direct channeling is only a scarce phenomenon for these TeOA membranes (otherwise back diffusion of $\mathrm{NO}_{3}^{-}$through the channels would outweigh the normal nitrate transport); - a reduction in carrier concentration in the LM-phase is compensated by a reduction in diffusion pathlength through the membrane (the membrane gets thinner by solvent loss).

Contrary to this the membrane with TOMA as a carrier shows an entirely different stability behaviour. Only at the start the flux is comparable to the case where the TeOA-membrane is used, but then it decreases drastically till after ca. 3 days the process of nitrate removal has practically stopped. It is obvious that this is due to removal of especially TOMA from the membrane. This agrees with the results of previous research described in Ref. [1] (Table 1), where it was found (using Accurel as support) that the carrier TOMA is removed to a much larger extent from the membrane than TeOA.

It is remarkable that the situation for which $\mathrm{CTF}>1$ is reached sooner for the TeOA-membrane having a more constant flux behaviour. As was stated before this point is reached when some water channels have been formed by the degradation mechanism. Therefore we have to conclude that the solvent is removed to a higher extent (or more localized) for the system with TeOA compared to that with TOMA. This agrees with the results described in Ref. [1] (Table1) where it was found that after 6 days more 0 -NPOE is removed from a membrane with TeOA as carrier than from a membrane with TOMA. This again can be explained by the very fast removal of the carrier TOMA from the membrane. The concentration of TOMA in the LM-solvent decreases to such an extent that the driving force for solvent-removal disappears and therefore the breakthrough (leakage) point is reached at a later time.

\section{Removal of $L M$-phase components}

\section{Removal of solvent}

Based on the experimental results described in the previous section, using decanol as solvent, the preliminary conclusion was drawn that the LM-phase is removed from the membrane by shearing forces excerted at the membrane interface by an aqueous phase with a low salt concentration. To verify this assumption identical aqueous phases were brought on both sides of the membrane and the amount of solvent removed was determined under different experimental conditions. This behaviour was also investigated for membranes with dibutylphtalate (DBP), dioctyladipate (DOA) and oNPOE as solvent to enable a further comparison with the instability results obtained in Ref. [1]. When using an SLM with decanol or DBP the aqueous phases were saturated with the solvent in question in the way described before to exclude solubility effects causing SLMinstability.

To simplify the system, these experiments were carried out in the absence of carriers in the solvents. To obtain measurable effects within reasonable times again Celgard was used as support. The experimental conditions (duration of the experiment, waterflow velocity and $\mathrm{NaCl}$ concentration) were chosen such that the instability could be measured accurately. In Fig. 3 the removal of the various solvents from the membranes is given as a function of the $\mathrm{NaCl}$ concentration in the aqueous phases. These 


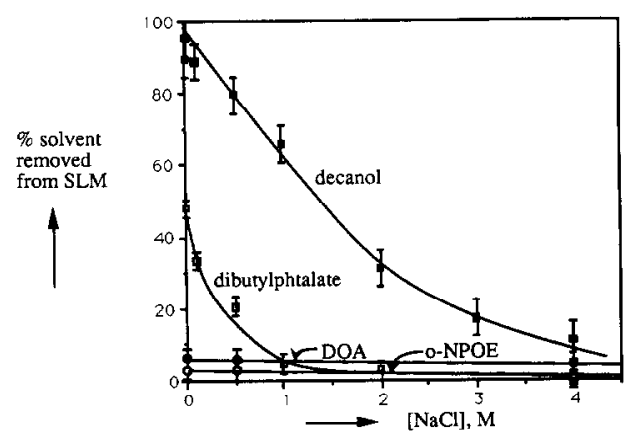

Fig. 3. Solvent removal from SLM after $7 \mathrm{hr}$ as a function of [ $\mathrm{NaCl}]$ of the aqueous phases; water flow rate: $5.5 \mathrm{ml} /$ sec; F- and S-side equal salt concentration.

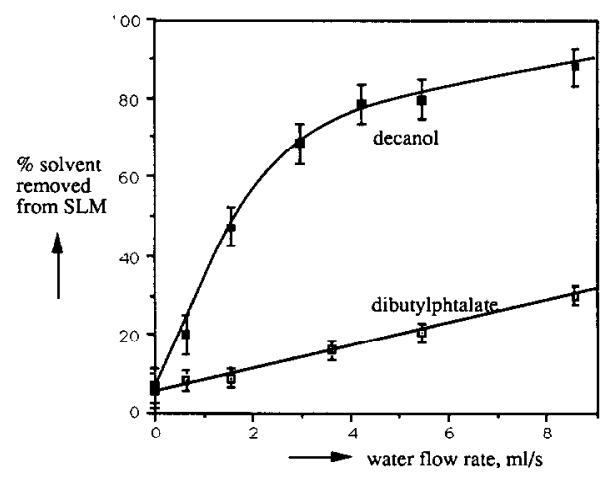

Fig. 4. Solvent removal from SLM after $7 \mathrm{hr}$ as a function of flow rate of the aqueous phases; $[\mathrm{NaCl}]_{\mathrm{F}}=[\mathrm{NaCl}]_{\mathrm{S}}=0.5$ M.

measurements were made after a period of $7 \mathrm{hr}$ and the flow velocity of the aqueous phases was $5.5 \mathrm{ml} / \mathrm{sec}$.

Figure 4 shows the removal of decanol and DBP from the membrane as a function of the water flow velocity. This behaviour has not been studied for DOA and 0 -NPOE because of the small amount of these two solvents removed from the membrane at a flow velocity of $5.5 \mathrm{ml} /$ sec.

Finally the removal of decanol as a function of time is examined and given in Fig. 5 .

From the results given in Figs. 3-5 it can be concluded that decanol and DBP are being removed gradually from an SLM and that the amounts removed increase with a decrease in

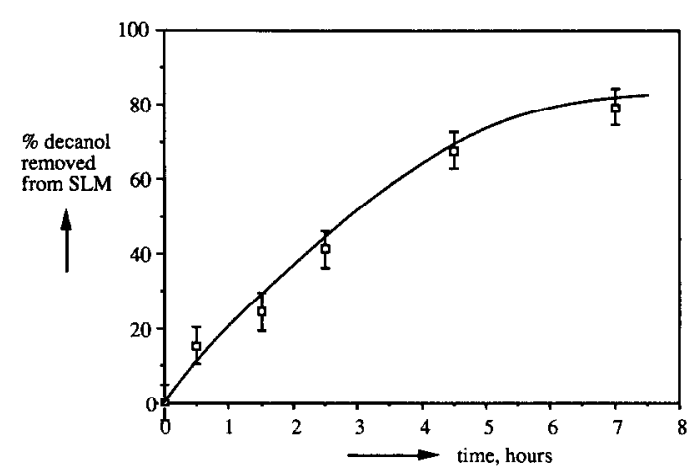

Fig. 5. Decanol removal from SLM as a function of time; $[\mathrm{NaCl}]_{\mathrm{F}}=[\mathrm{NaCl}]_{\mathrm{S}}=0.5 \mathrm{M}$; water flow rate: $5.5 \mathrm{ml} / \mathrm{sec}$.

the salt concentration of the aqueous phases and with an increase in their flow velocities. This result is in agreement with the results of the membrane failure experiments and Ref. [1]. It shows why, in Ref. [1], with a stripping phase of $0.5 \mathrm{M} \mathrm{NaCl}$, more decanol and DBP is removed from the membrane than using $4.0 \mathrm{M}$ $\mathrm{NaCl}$. Furthermore it explaines why the DBPmembrane failed at a composition of $0.5 \mathrm{M}$ $\mathrm{NaCl}$ in the stripping phase and stayed intact with $4.0 \mathrm{M} \mathrm{NaCl}$.

On the other hand we find that DOA and oNPOE are hardly removed from the membrane, even with low salt concentrations in the water phase. This is in agreement with the results given in Ref. [1], in which the use of these two solvents leads to relatively stable SLMs.

These results clearly show that the instability of SLMs is not caused by the presence of an osmotic pressure difference over the membrane: there simply is no osmotic pressure difference operative during our experiments.

\section{Removal of carrier}

During the long term permeation experiments described before we found that the carrier TOMA was removed much faster from the membrane than TeOA. When shaking aqueous phases with LM-phases in a test tube sometimes relatively stable emulsions are formed, depending on the composition of the phases. In 
all cases more LM-phase is being emulsified and more stable emulsions are formed in the presence of TOMA than in the presence of TeOA. Based on these data the hypothesis arises that SLM-instability does result from the formation of stable emulsions. We also found that less stable emulsions were formed when the salt concentration in the aqueous phases was increased. To test the hypothesis that emulsion formation causes SLM-instability, the influence of the composition of the aqueous phases on SLM-stability has been determined. Like before completely identical compositions were chosen for the feed and stripping phases at both sides of the membrane.

At first the aqueous phases were analysed for the presence of carrier. Due to adsorption of the carrier to the glass-parts of the apparatus these measurements were time dependent and therefore irreproducible. Because an accurate determination of the carrier was impossible in this way it was decided to study the removal of carrier by analysing the residual carrier content in the SLMs itself. A disadvantage is that this method is destructive which means that for the determination of the leaching out behaviour as a function of time different membranes had to be tested. In these experiments the conditions again were chosen such that the instability effects were well measurable.

Results. In Fig. 6(a) the removal of the carriers TOMA and TeOA (from LM-phases with $o$-NPOE as solvent) is given as a function of the $\mathrm{NaCl}$ concentration in the aqueous phases. For the sake of clearness the curves representing the system with TeOA as a carrier are plotted bold-faced.

Because of the steep change in TeOA removal when aqueous phases with very low salt concentrations are used, we examined carrier removal from the membrane in more detail in the region of these low salt concentrations. The results are given (on a logaritmic scale) in Fig. 6(b). In these experiments $\mathrm{NaCl}$ solutions as
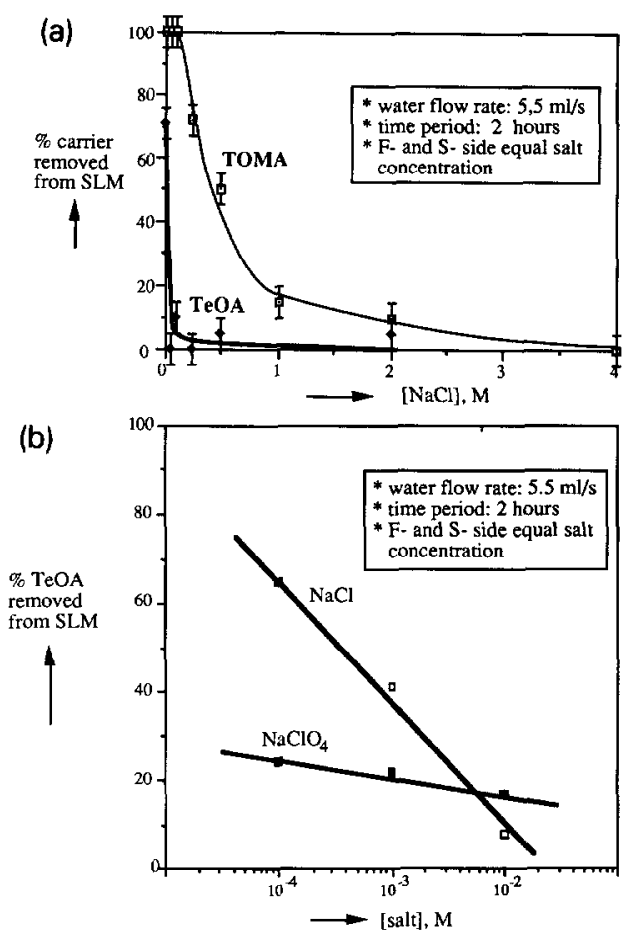

Fig. 6. (a): Carrier removal as a function of $\mathrm{NaCl}$ concentration in the aqueous phases and carrier structure; (solvent: 0 -NPOE). (b) Removal of TeOA (from LM-phases with $o$-NPOE as solvent) as a function of type of salt and salt concentration.

well as $\mathrm{NaClO}_{4}$ solutions were used as the aqueous phase.

To obtain the mass of the LM-phase removed (i.e. carrier and solvent) the weight of the membranes was determined before and after the experiments. The mass of the carrier removed was calculated from spectrophotometric analysis of the remaining LM-phase. The quantity of $o$-NPOE which had been removed from the membrane was obtained from these values. Based on these data the relative amounts of carrier and solvent removed are calculated as a function of the salt concentration. In Table 2 these data are given for the experiments in which $\mathrm{NaCl}$ solutions were used.

Figure 7 shows the carrier removal from the membrane as a function of the flow velocity of 
TABLE 2

Relative removal of carrier and solvent from SLMs for mall $\mathrm{NaCl}$ concentrations (equal salt concentration at $\mathrm{F}$ - and $\mathrm{S}$ side)

\begin{tabular}{lllll}
\hline $\begin{array}{l}{[\mathrm{NaCl}]} \\
(M)\end{array}$ & $\begin{array}{l}\text { Total } \\
\text { LM-phase } \\
\text { removed } \\
(\mathrm{mg} \pm 0.2)\end{array}$ & $\begin{array}{l}\text { Removal } \\
\text { of } \\
\text { carrier } \\
(\mathrm{mg})\end{array}$ & $\begin{array}{l}\text { of } \\
\text { o-NPOE } \\
(\mathrm{mg})\end{array}$ & $\begin{array}{l}\text { Larrier content } \\
\text { of removed } \\
\text { LM }\end{array}$ \\
\hline $10^{-4}$ & 1.7 & 1.5 & 0.2 & $73-100$ \\
$10^{-3}$ & 1.2 & 0.9 & 0.3 & $59-100$ \\
$10^{-2}$ & 0.9 & 0.2 & 0.7 & $6-43$ \\
\hline
\end{tabular}

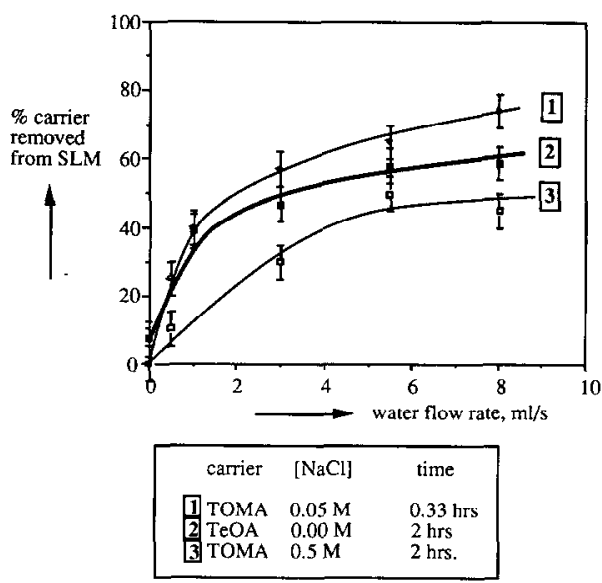

Fig. 7. Carrier removal from LM-phases with $o-\mathrm{NPOE}$ as solvent as a function of flow rate of aqueous phases.

the feed and the stripping phases for a number of cases with varying salt concentrations in the aqueous phases and at different measuring times.

Figure 8 shows the removal of carrier from the membrane in relation to the measuring time for different salt contents in the aqueous phases.

Figures 6(a) and 6(b) show that the removal of carrier from the SLM depends on the nature of the carrier, the salt concentration in the aqueous phases and the nature of the salt used. An increase in $\mathrm{NaCl}$ concentration in the water phases leads to a decrease in the percentage of carrier that is removed from the membrane. Furthermore from Figs. 8 and 7 it follows that

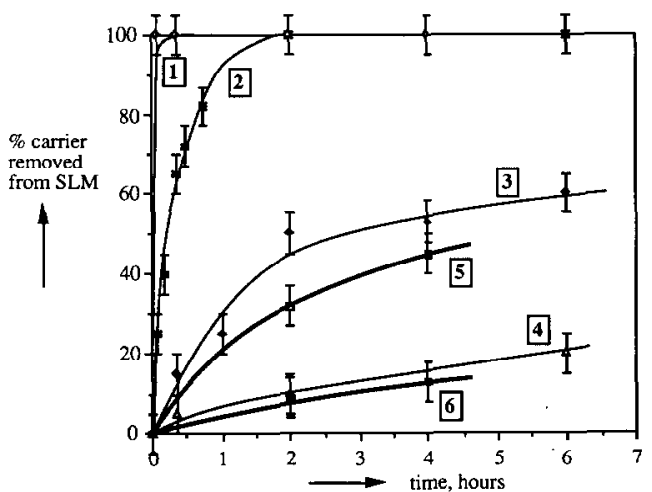

Fig. 8. Carrier removal as a function of time; carrier TOMA (curves 1-4), TeOA (Curves, 5,6); solvent o-NPOE; waterflow $5.5 \mathrm{ml} / \mathrm{sec}$; [Nacl] : $0.000 M(1), 0.05 M(2), 0.5 M$ (3), $2.0 M(4), 0.001 M(5), 0.01 M(6)$. Salt concentration on $\mathrm{F}$ - and $\mathrm{S}$-side equal.

this removal increases gradually with time (until complete removal is reached) and increases when enhancing the flow velocity of the aqueous phases.

From the various figures it is evident that under comparable conditions the carrier TOMA is removed much faster from the membrane than TeOA. The large increase in TeOA removal at high salt dilutions $\left(10^{-2}-10^{-4} M\right.$ $\mathrm{NaCl}$ ) is quite remarkable. Even more remarkable is the influence which $\mathrm{ClO}_{4}^{-}$ions in the aqueous phases have on the stability of the membrane (see Fig. 6 b). Even extremely low concentrations of this type of salt $\left(10^{-4} M\right)$ suppress the removal of the carrier from the membrane quite effectively.

With these rather simple laboratory experiments a rapidly applicable method has been developed which can provide relevant information on the influence of various stability factors of our SLMs. Perhaps this method is also applicable for other SLM-systems so that it can provide a prediction (on short term) for the long term permeability and stability behaviour of the membranes.

Considering the correlation between the formation of emulsions, in test tube shaking ex- 
TABLE 3

Calculated average size of emulsions droplets removed from TeOA/o-NPOE membranes by a lateral flow of $\mathrm{NaCl}$ solutions

\begin{tabular}{ll}
\hline $\begin{array}{l}{[\mathrm{NaCl}]} \\
(M)\end{array}$ & $\begin{array}{l}\text { Diameter of droplets removed } \\
\text { from SLM }(\mathrm{nm})\end{array}$ \\
\hline $10^{-4}$ & $2-8$ \\
$10^{-3}$ & $2-11$ \\
$10^{-2}$ & $20-140$ \\
\hline
\end{tabular}

periments, and the removal of carrier from the membrane, these facts reinforce the hypothesis that formation of emulsion causes the SLMdegradation.

The results given in Table 2 can also be used to get a rough estimation of the size of the emulsions droplets which are removed from the membrane for the system with TeOA/o-NPOE as LM-phase. Assuming that the concentration of carrier in the bulk of the emulsion droplet is equal to the concentration in the bulk of the liquid membranes $(0.2 \mathrm{M})$ and that the skin of the spherical emulsion droplet consists of a close packed monolayer of TeOA-molecules with a cross-sectional diameter of $0.8 \mathrm{~nm}$ [2] the following average emulsion droplet diameters can be calculated.

The relative errors in Table 3 are large because the relative error in the determination of the carrier content of the removed LM-phase was rather large and the calculation of the size of the emulsion droplets is very sensitive for this quantity.

\section{Emulsion formation and stability}

As already mentioned before, shaking an LMphase with an aqueous phase leads to a more or less turbid emulsion phase, depending on the composition of the phases. This occurs for LMphases containing carriers as well as for pure solvents. Studied microscopically, the emulsions appear to contain moving small spheres. Depending on the preparation method these emulsions droplets have sizes varying from 0.1 $\mu \mathrm{m}$ to tens of micrometers (as determined with the Mastersizer).

The emulsions were characterized by measuring the zeta-potential of the droplets. The stability behaviour of the emulsions was quantitatively studied by turbidity measurements as a function of time. For these measurements, in the systems without carrier, decanol was chosen as the organic phase because this solvent provides the largest instability effects, also depending on the salt content of the aqueous phase. A comparison with other pure solvents is hard to make because of the influence of the specific gravities on the light transmission at the time of the measurements. For a correlation with previous experiments the LM-system with o-NPOE as solvent and TOMA or TeOA $(0.2 M)$ as carrier has also been studied.

\section{Zeta-potential}

The results of the electrophoretic measurements for a number of systems emulsified in double distilled water are given in Table 4.

\section{Light transmission measurements}

Decanol. The light transmission as a function of time for $50 \mathrm{ml}$ aqueous phase with different salt content after 3 min of stirring with three droplets of decanol is plotted in Fig. 9.

In all cases the same trends were observed. An increase in salt content leads to more rapid clarification of the emulsions. However the reproducibility of these measurements is low.

\section{TABLE 4}

Zeta-potentials for emulsions of LM-phases in double-distilled water

\begin{tabular}{ll}
\hline Emulsified LM-phase & Zeta-potential $(\mathrm{mV})$ \\
\hline decanol & -33 \\
TeOA/a-NPOE & +41 \\
TOMA/o-NPOE & +58 \\
\hline
\end{tabular}




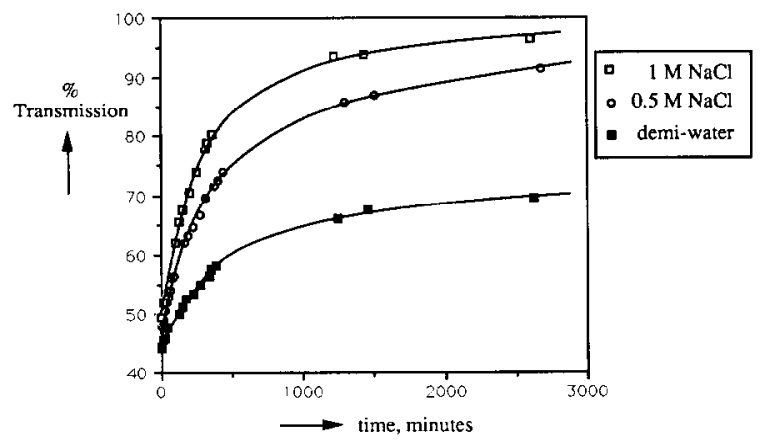

Fig. 9. Light transmission of decanol emulsions (obtained by stirring) as a function of time and $\mathrm{NaCl}$-concentration.

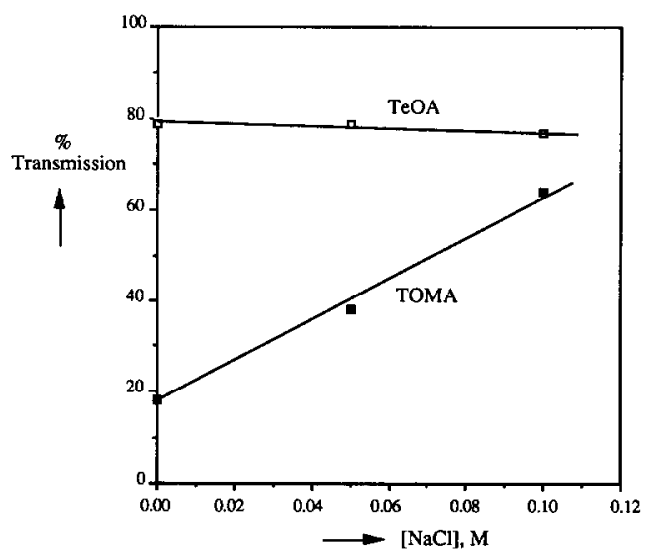

Fig. 10. Light transmission of emulsions (obtained by shaking) as a function of $\mathrm{NaCl}$ concentration and type of carrier; solvent $o$-NPOE.

LM-phase with carrier. The behaviour of emulsion formation of 0 -NPOE containing carrier is studied by shaking $1 \mathrm{ml}$ organic phase with $3 \mathrm{ml} \mathrm{NaCl}$ solution in a spectrophotometer tube. The transmission of the resulting emulsion after $5 \mathrm{~min}$ as a function of $\mathrm{NaCl}-$ content is given in Fig. 10. At low salt concentrations the emulsions with TOMA as carrier were very stable. Even after several months no coalescence of the droplets was observed.

$L M$-phase with TeOA as carrier. It is clear from previous measurements that almost no emulsion formation took place of the LM-phase with TeOA as carrier under given conditions. Since it was assumed earlier that the removal

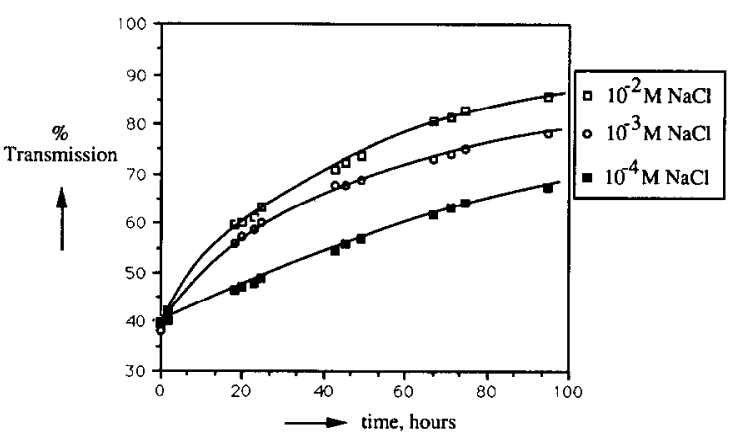

Fig. 11. Light transmission of TeOA 10 -NPOE emulsions (obtained by ultrasonic vibration) as a function of time and $\mathrm{NaCl}$-concentrations.

of this carrier from the membrane by shearing with aqueous phases having a very low salt content could also be ascribed to the formation of emulsions, a more powerful method (ultrasonic vibration) has been used to prepare emulsions of this LM-phase. By diluting the so formed emulsions using aqueous solutions with different salt content the stability behaviour of the emulsions could be studied as a function of salt content under comparable starting conditions. In this way the problem of the poor reproducibility of emulsion formation by shaking or stirring is avoided and the results can be compared also for different types of salts in the aqueous phases. An example of the change in transmission as a function of time when the $\mathrm{NaCl}$ content is varied is given in Fig. 11.

As an indirect measure for the instability of the emulsions we used the clarification factor. This value can be determined from the slope of the light transmission curve extrapolated to short times ( $\Delta \%$ transmission/time). This factor is plotted in Fig. 12 as a function of the salt content in the aqueous phases.

\section{Discussion of emulsion stability measurements}

Table 4 shows that in the three systems studied emulsions are present which are stabilized by charge. The negative zeta-potential for the 


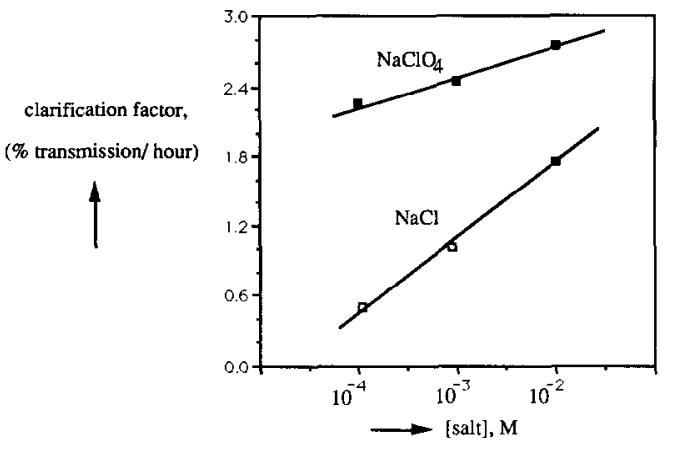

Fig. 12. Clarification factor at $t \rightarrow 0$ for TeOA/o-NPOE emulsions as a function of type of salt and its concentration is aqueous phases.

decanol emulsions is probably caused by the adsorption of negative ions at the oil/water interface. Usually the anions will be somewhat more oil soluble and adsorbable than the cations, so the oil droplets should have a net negative charge [4].

For the systems with carrier the positive zetapotential is explained by the adsorption of positively charged quaternary ammonium ions at the droplet interface. The larger zeta-potential of emulsions with TOMA as carrier agrees with the more surface active behaviour of this carrier in comparison with TeOA.

From Figs. 9-11 it follows that for all three systems mentioned the turbid emulsions become transparant more rapidly for increasing $\mathrm{NaCl}$ concentration. This is caused by the creaming or sedimentation of coalesced emulsions droplets. The decreasing stability of the emulsions when increasing the salt content is explained by the "DLVO-theory" [5] which describes the stability of colloidal systems. Charge stabilized emulsion droplets encounter Londen-van der Waals attraction forces and electrostatic repulsion forces by the overlap of the electrical double layer of the droplets. The zeta-potential, the potential at the hydrodynamic shear plane, is a measure for the repulsion energy and so for the stability of the emulsions. By increasing the salt content this electrical double layer is getting thinner and the repulsion forces of the emulsions droplets will decrease by which their stability decreases.

The stationary velocity $v$ by which a stabilized emulsion droplet creams or sediments due to the difference in density with the continuous phase, is given by [6]:

$v=\Delta d g r^{2} 2 / 9 \eta$

In this formula $\eta$ is the viscosity of the medium, $g$ the acceleration due to gravity, $r$ the radius of the emulsion droplet and $\Delta d$ is the difference in density between the droplet phase and the water phase. From this equation it follows that by increasing the radius of the emulsion droplets, due to coalescence, the emulsions will cream or sediment more rapidly and so they will clarify more rapidly.

Because decanol has a density of 0.83 coalesced decanol emulsions will cream. When studying the influence of the salt content on the creaming of these emulsions a correction had to be made for the increase in the density difference when increasing the salt content. The effect of this correction is rather small (e.g. 17\% for $[\mathrm{NaCl}]=0.5 M$ compared with double-distilled water) as compared with the differences in the turbidity of the decanol emulsions (see Fig. 9, almost a factor of 2), and therefore it can be concluded that the stability of decanol emulsions does decrease when increasing the $\mathrm{NaCl}$ concentration.

The LM-phases with $o$-NPOE as solvent and TOMA or TeOA as carrier have a density of $1.03 \mathrm{~g} / \mathrm{ml}$. Because aqueous solutions with a $\mathrm{NaCl}$ concentration lower than $10^{-2} M$ have densities below $1.0005 \mathrm{~g} / \mathrm{ml}$, the coalesced emulsion droplets will sediment. The increase in density of the aqueous phases with these very small salt concentrations is so minute that it has no measurable influence on the behaviour of sedimentation. So here also we can conclude that for increasing $\mathrm{NaCl}$ concentrations the emulsions are getting less stable. 
The irreproducibility in the formation of decanol emulsions when stirring the organic/ aqueous phases is quite normal for these type of systems. It correlates with the fact that emulsification is a complicated process and that this process until now is not very well understood as was expressed by Walstra [7] in the following way: "Unfortunately, much knowledge about emulsification is still of a cookbook nature."

From Fig. 10 it follows that the LM-phase with TOMA as carrier is emulsified much better than using TeOA. This is caused by the differences in geometric structure of the carriers. It is known from literature that the structure of the surfactant plays an important role when forming emulsions. Generally a surfactant dissolved in an organic solvent is less surface active when the hydrophilic group is placed more in the centre of the molecule. For our system this means that the TOMA-molecule having one methylgroup is much more surface active than the TeOA-molecule with its symmetrical structure of four octyl chains.

Apart from the structure of the carrier molecule the type of counter-ion is also of importance for the stability of emulsions, as is shown in Fig. 12. The emulsions with perchlorate ion as a counter ion had a much higher clarification rate than with chloride ion at equal salt concentrations. Two possible explanations for this are:

- because the perchlorate ion is larger than the chloride ion, it will show more specific adsorption, by which it will have a larger effect on the charge compensation;

- the perchlorate ion is less hydrated as compared to the chloride-ion (according to the Hofmeister series, see Ref. [2]). Therefore the perchlorate ion has a stronger interaction with the ammonium cation at the interface compared to the chloride ion.

For both reasons the electrical double layer of the emulsion droplet with perchlorate ions will be thinner and the repulsion energy will be lower which results in less stable emulsions.

\section{SLM-degradation by emulsion formation}

We want to point out here that we have three clear examples of a direct correlation between membrane instability (solvent and carrier removal run parallel with membrane instability) and emulsion stability of the LM-phase dispersed in aqueous solutions. These examples are:

(a) Comparing Figs. 3 and 9 for decanol as LMphase (without carrier) it can be seen that an increasing salt content in the aqueous phases decreases the solvent removal from SLMs, hence enhances membrane stability (Fig. 3), while the stability of decanol emulsions is decreased (transmission enhanced at higher salt concentration, see Fig. 9).

(b) A similar correlation is found for the salt effect on carrier removal and emulsion stability for the system with $o-N P O E$ as solvent and TeOA or TOMA as carrier, Figs. 6(a) and 10. Especially for TOMA the high level of carrier removal at low salt concentration, giving a very instable membrane (Fig. 6a) correlates strongly with a low level of light transmission indicating a stable emulsion at low salt content (Fig. 10). On the other hand the system with TeOA as carrier shows for all $\mathrm{NaCl}$ concentrations, except for extremely low values, both a small percentage of carrier removal (Fig. 6a), hence more stable membranes, and a high light transmission for the emulsions (Fig. 10), i.e. instable emulsions.

(c) Finally a comparison between Figs. 6(b) and 12 for the system with $o$-NPOE/TeOA as LM-phase and very low salt concentrations in water provides the same correlation as above between a decrease in carrier removal (Fig. 6b) and an increase in emulsion clarification (less stable emulsions) for the higher salt content. Furthermore the most stable SLMs are formed 
for the system with perchlorate as counter-ion, which has the highest emulsion clarification factor (least stable emulsions).

In view of this significant similarity between the stability of emulsions and the instability of SLMs the conclusion is justified that instability of SLMs is caused by the loss of emulsion droplets from the LM-phase into the aqueous phase.

For the sake of completeness it is put forward once more that a correlation of any importance is not found between the instability of SLMs and other physical parameters; e.g. the relation between the SLM-instability and the lowering of the interfacial tension is not unambigious (as was already mentioned in Ref. [1]). The large influence of the salt content on the removal of LM-phase from the membrane is not at all reflected in the change in interfacial tensions between the LM-phase and the aqueous phases (see Ref. [1]).

Molecular solubility of components from the LM-phase into the aqueous phase can also be excluded as a cause for these instability effects since neither the carrier TeOA nor solvent $o$ NPOE do show any substantial solubility in the aqueous phase. In contrast it was shown from the experiments described in our study that they do form emulsions when mixed thoroughly with the aqueous phases.

The interfacial tensions between the different LM-phases and the aqueous phase [1] are so large that the instability effects of the SLMs can not be attributed to spontaneous formation of emulsions. It is evident that, when in a certain system emulsions could form spontaneously between the LM-phase and an aqueous phase it would undoubtedly lead to notorious SLM-instability effects.

A mechanism which does agree with the instability phenomena observed, is that local deformations of the liquid meniscus in the pore of the support may lead to the splitting off of emulsion droplets. The roughly calculated sizes of emulsion droplets removed from the system with TeOA/o-NPOE as LM-phase (Table 3), show that these emulsion droplets are small enough that only a part of the meniscus has to be deformed. This means that for a pore diameter of ca. $200 \mathrm{~nm}$ and a droplet size from 4 $80 \mathrm{~nm}$ only ca. 2 à $40 \%$ of the width of the meniscus has to be deformed. Such local deformations are given schematically in Fig. 13.

These deformations can originate from one of the following sources:

- "Kelvin-Helmholz instabilities": these instabilities arise when two phases move with different velocities parallel to the interphase [8]. It causes, for instance, waves to develop on a water surface over which the wind blows.

- vibration of the membrane; because the membrane is not supported by a rigid screen it is repeatedly deformed a bit due to the pulsating aqueous phases. These vibrations can cause formation of ripples on the surface of the LMphase, because any disturbance of the interface will cause waves to develop [7].

The next step then is to explain how these ripples at the interface can lead to the formation of droplets which were found to be the cause of membrane degradation. A possible explanation is the formation of interfacial tension gradients which result in Marangoni effects. If the interface is locally curved so that the concave side is the phase that provides the surfactant, the curved part will have a higher interfacial tension, since it receives the smallest quantity of surfactant molecules per unit surface area. Hence interfacial transport of surfactant and a flow of liquid dragged along towards the point of the strongest curvature will occur leading to

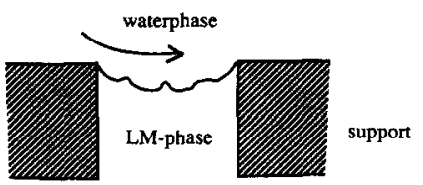

Fig. 13. Local deformations of the meniscus of the LMphase in the pore of the support. 
an instable situation. This may well cause droplet shredding. However, the action of dynamic interfacial effects forms a formidable problem that is far from being understood [7].

That we are dealing with Marangoni effects is also indicated by the very small sizes of the emulsion droplets removed from the membrane (Table 3). The formation of emulsion droplets, as a consequence of Marangoni effects, with diameters varying from 10 to $50 \mathrm{~nm}$ has for instance been published earlier [4].

Table 3 shows that on decreasing the salt content in the water phases also the size of the emulsion droplets which are removed from the membrane decreases. So, very few relatively large emulsion droplets are removed from the membrane for a salt content of $10^{-2} \mathrm{M} \mathrm{NaCl}$ in the aqueous phases, while many small emulsion droplets are removed for $10^{-3} \mathrm{M} \mathrm{NaCl}$ and $10^{-4} \mathrm{M} \mathrm{NaCl}$. These results will be confirmed by experimental results described in Ref. [9]. In other words a larger number of energetically unfavourable, small emulsions droplets are formed as they are stabilized better.

From Fig. 10 it followed that under identical conditions an LM-phase with TOMA as carrier is emulsified to a larger extent than an LMphase with TeOA as carrier. Because of the better stability of emulsions with TOMA as surfactant, emulsion droplets which are removed by lateral forces exerted on an SLM with TOMA as carrier will in the average be smaller than with $\mathrm{TeOA}$ as carrier. This explains why more solvent is removed from the membrane with $\mathrm{TeOA}$ as carrier, which results in membrane leakage at an earlier time, as compared to the membrane with TOMA as a carrier.

An emulsion droplet which is split off can coalesce again immediately with the "parent"phase. Coalescence is probably of importance for droplets that have just been formed and have not yet acquired an equilibrium adsorption layer [7]. It is clear that stable emulsion droplets, with a fully developed electrical double layer, will coalesce much less readily than emulsion droplets with a lower stability. This explains also the similarity between the stability of emulsions and the instability of SLMs.

The considerations just given are based on reference points found in the literature with respect to emulsion formation. However, it shows that there is still much obscure with regards to the mechanism of emulsion formation and the rate of coalescence. For that reason it is impossible at this moment to formulate and proof the hypothesis of emulsion formation at an SLM interface more rigorously.

\section{Final remarks on the degradation mechanism}

Removal of LM-phase will force the meniscus of the LM-phase to withdraw in the pore of the support. Water will be filling up the volume in the pore (see also Ref. [1]). Eventually the meniscus of the LM-phase will reorientate and be found again at one of the boundaries of the membrane. This could occur spontaneously due to the fact that there are gradients in pore diameters in the support. The smallest pores of the support are present at the membrane surface [10].

When spontaneous transport of LM-phase to the interface is hindered by bottle necks in the pore structure the transport might be promoted by vibrations of the membrane. Another driving force for transport of LM-phase to the interface of the membrane could be small pressure differences over the SLM. The pressure differences are "infinitesimal" in the experiments described here. But it is known [11] that pressure differences over the membrane as low as $0.02 \times 10^{5} \mathrm{~Pa}$ can increase instability effects of an SLM. When the meniscus of the LMphase contacts again the water phase which flows along the membrane, the formation of emulsion droplets will continue according to the mechanism described above. 


\section{Conclusions}

The results discussed in this paper show that the instability of SLMs does not result from osmotic pressure differences or spontaneous emulsification. It is clear that the structure of the carrier, the salt content and the type of the counter ions in the aqueous phase influence emulsion stability and SLM instability in parallel fashion. Therefore we propose a mechanism for the degradation of SLMs in which shear induced break away of emulsion droplets at the feed interface plays the main role.

For the development of stable SLMs the composition of the LM-phase has to be chosen in such a way that it has little capacity for the formation of emulsions. Especially the structure of the carrier is important. Furthermore the instability effects can be diminished by varying the composition of the aqueous phases, although in practice the boundary conditions are determined by the total process occurring in the SLM-system.

Finally the type of experiments studied extensively in this paper (in which the removal of solvent and carrier is followed by flowing identical aqueous phases parallel to the membrane surface) are very suitable for an investigation into the influence of the composition of the phases on SLM-stability. In this way the influence of various parameters on the long term permeability of the membranes can be predicted using short term experiments.

\section{Acknowledgement}

The authors wish to thank G. Spijkerman and C. Kokkeler for the experimental help and the stimulating discussions. This work was financially supported by the Dutch Ministry of Economic Affairs, within the Dutch research program on membranes.

\section{References}

1 A.M. Neplenbroek, D. Bargeman and C.A. Smolders, Supported liquid membranes: instability effects, J. Membrane Sci., 67 (1992) 121.

2 A.M. Neplenbroek, D. Bargeman and C.A. Smolders, Nitrate removal using supported liquid membranes: stransport mechanism, J. Membrane Sci., 67 (1992) 107.

3 M. Tromp, M. Burgard, M.J.F. Leroy and M. Prevost, Extraction of gold and silver cyanide complexes through supported liquid membranes containing macrocyclic extractants, J. Membrane Sci., 38 (1988) 295.

4 A.W. Adamson, Emulsions and foams, in: Physical Chemistry of Surfaces, Interscience, New York, NY, 1967, Chap. 11.

5 (a) B.V. Derjaguin and L. Landau, Theory of the stability of strongly charged lyophobic sols and of the adhesion of strongly charged perticles in solutions of electrolytes, Acta Physiochem. USSR, 14 (1941) 633. (b) E.J.W. Verwey and J.Th.G. Overbeek, Theory of the Stability of Lyophobic Colloids, Elsevier, Amsterdam, 1948.

6 W.J. Beek and K.M.K. Muttzall, Transport Phenomena, Wiley, New York, NY, 1975.

7 P. Walstra, Formation and emulsions, in: P. Becher (Ed.), Encyclopedia of Emulsion Technology, Vol. 1, Marcel Dekker, New York, NY, 1983, Chap. 2, pp. 57127.

8 S. Chandrasekhar, Hydrodynamic and Hydromagnetic Stability. Clarendon, Oxford, 1961, Chaps. 1012.

9 A.M. Neplenbroek, D. Bargeman, C.A. Smolders, Supported liquid membrane. Stabilization by gelation, J. Membrane Sci., 60 (1991) 00.

10 T. Sarada, L.C. Sawyer and M.I. Ostler, Three dimensional structure of Celgard microporous membranes, J. Membrane Sci., 15 (1983) 97.

11 H. Takeuchi, K. Takahashi and W. Goto, Some observations on the stability of supported liquid membranes, J. Membrane Sci., 34 (1987) 19. 